\title{
L'animation théâtrale, une préhistoire du théâtre pour les jeunes
}

Theatrical Animation, a Prehistory of the Theater for Young People

\section{Sibylle Lesourd}

\section{OpenEdition}

\section{Journals}

Édition électronique

URL : https://journals.openedition.org/recherchestravaux/783

DOI : $10.4000 /$ recherchestravaux.783

ISSN : 1969-6434

Éditeur

UGA Éditions/Université Grenoble Alpes

Édition imprimée

Date de publication : 1 décembre 2015

Pagination : 85-95

ISBN : 978-2-84310-314-8

ISSN : 0151-1874

\section{Référence électronique}

Sibylle Lesourd, «L'animation théâtrale, une préhistoire du théâtre pour les jeunes », Recherches \& Travaux [En ligne], 87 | 2015, mis en ligne le 01 janvier 2017, consulté le 29 octobre 2021. URL : http:// journals.openedition.org/recherchestravaux/783 ; DOI : https://doi.org/10.4000/recherchestravaux. 783 
Sibylle Lesourd

Université Paris Sorbonne

\section{L'animation théâtrale, une préhistoire du théâtre pour les jeunes}

Durant l'été 1970, la troupe des marionnettes de Turin (futur Teatro dell'Angolo) est chargée par la municipalité d'organiser quelques spectacles pour enfants dans les Parcs Robinson. Ces jardins publics sont particulièrement fréquentés, l'été, par les enfants des familles défavorisées ne pouvant se permettre de vraies vacances hors de la ville. Or les jeunes spectateurs assistent aux spectacles avec un enthousiasme modéré, sauf à la fin de la représentation lorsque, le rideau enfin tombé, ils peuvent à leur tour s'emparer des marionnettes. Face à un tel constat, les artistes décident de confier d'emblée les marionnettes aux jeunes afin qu'à travers des histoires "apparemment déglinguées et grossières", ils en viennent à «se raconter eux-mêmes ${ }^{\mathrm{I}}$. Ainsi va naître l'animation théâtrale.

En France et en Italie, le rôle de l'animation dans l'émergence du théâtre pour les jeunes nous semble déterminant. À partir des années 1960-I970, des artistes se sont demandé jusqu'à quel point leur travail de création pouvait être mené en collaboration avec l'enfant. Faut-il, lorsqu'on entend créer en direction de la jeunesse, s'interroger sur les mécanismes et les contenus de la psychologie enfantine? Doit-on même emprunter à l'enfant ses propres idées? Engageant une certaine foi dans les potentialités créatives des plus jeunes - fondée sur le respect de leur personne et parfois sur ce qu'on pourrait appeler un mysticisme de l'enfance - ces artistes ont recherché l'immersion dans les espaces où pouvait s'exprimer la culture enfantine, au premier rang desquels figurait l'école. Inventée par Catherine Dasté, largement diffusée en Italie du Nord, l'animation théâtrale a pu apparaître comme une «méthode miracle»; recueillant directement à la source enfantine les matériaux propices à la création,

I. Cité dans G.-R. Morteo, L. Perissinotto et al., Animazione e città, Turin, Musolini Editore, 1980, p. 20 : «In quelle storie, per quanto sgangherate e rozze, i bambini raccontavano se stessi.» 
elle conférait un gage d'authenticité à un théâtre pour l'enfance qui n'inspirait jusque-là que suspicion. En vérité, l'engagement des artistes dans l'animation préfigurait la naissance de formes esthétiques nouvelles destinées à l'enfant spectateur. De Dasté à Rodari, en passant par les expériences du Teatro del Sole et du Teatro Gioco Vita, les méthodologies ont différé mais l'animation s'est toujours fondée sur une collusion féconde entre pratique créative avec les jeunes et invention dramatique à leur intention.

\section{La «méthode» Dasté et ses avatars}

En France, Catherine Dasté, de 1960 à 1977, est la première à faire de l'imagination des enfants un matériau poétique essentiel à l'élaboration de ses spectacles. Elle trace un chemin inédit qui inspirera les créateurs qui lui succéderont, sinon dans la démarche, du moins dans l'esprit qui l'anime : il est impossible après elle d'éluder toute réflexion relative à la spécificité de l'imaginaire enfantin lorsqu'on crée du théâtre pour les jeunes. Pour Les Musiques magiques, premier spectacle avec les Tréteaux de Saint-Étienne en I96I, la méthodologie retenue est assez simple : les artistes décident de recueillir au magnétophone les histoires que racontent spontanément les élèves... Or le succès en est retentissant et la méthode Dasté fait couler de l'encre. Son pouvoir de fascination provient du fait qu'elle s'inscrit parfaitement dans la mythologie de l'enfant créateur qui triomphe dans la deuxième moitié $\mathrm{du} \mathrm{Xx}^{\mathrm{e}}$ siècle : avec la foi de Peter Pan, la critique salue les «vingt-cinq dramaturges en culottes courtes ${ }^{2}$ » et poétise à l'envi l'expérience de Catherine Dasté, qu'on imagine se promenant «magnétophone en bandoulière, et [avec] un groupe de gosses qui rêvent tout haut ${ }^{3} »$. Au cours de son expérience, Catherine Dasté découvre que l'imagination enfantine est foncièrement différente de l'imagination adulte. Cette hétérogénéité de nature éclate dans le processus d'invention d'histoires. Plus elle écoute parler les enfants, plus elle est sensible à l'élaboration spontanée d'images symboliques qui semblent chargées de significations inconscientes et créent une forte impression poétique, proche du surréalisme. Un garçon d'une classe de Ménilmontant lui a fait, par exemple, le récit suivant :

Voilà M'dame - il y avait une dame qui avait eu un bébé. Elle l'a mis dans son berceau; mais elle a oublié de le sortir. Et quand il a eu trente ans, il était toujours

2. Le Monde, 24-25 mars 1968.

3. "Entretien avec Catherine Dasté» par le Père Auguste, conservé dans les archives du Théâtre de la Pomme Verte (Société d'histoire du théâtre). 
dans le berceau. Alors ses jambes dépassaient et il était serré. Il était mal mais elle oubliait de le sortir. - Voilà M'dame, c'est tout ${ }^{4} \ldots$

Catherine Dasté apprend à reconnaître ces surgissements métaphoriques qui contrastent vivement avec l'environnement imaginaire assez conventionnel au sein duquel ils se produisent : des récits de voyage, avec des péripéties merveilleuses... S'il existe une pensée enfantine originale, distincte dans son contenu de la pensée adulte - ce que soutenait déjà un psychologue comme Jean Piaget -, il est essentiel à ses yeux d'en capter la richesse et de rejeter avec rigueur toute influence extérieure susceptible de l'altérer. À cet égard, l'invasion grandissante de la télévision est vécue par Catherine Dasté comme un fléau pour l'imaginaire qui troque ses contenus personnels contre des contenus stéréotypés. L'éducation traditionnelle, elle aussi, fait barrière à l'inventivité : tout en acquérant des capacités rationnelles, l'enfant en vient peu à peu à juguler ses fantasmes. Pour sélectionner la matière de ses spectacles, Catherine Dasté se fie donc à son intuition qui saisit une différence qualitative dans certains éléments des récits enfantins. Car il s'agit bien de discriminer : dans les récits des petits, tout n'est pas bon à prendre. Ici le mysticisme se teinte de pragmatisme.

Sa démarche expérimentale se situe quelque part entre ethnologie et psychanalyse. La diffusion toute récente des travaux de Claude Lévi-Strauss incite en effet à s'ouvrir aux enfants à la façon d'un chercheur qui «observe et écoute une ethnie inconnues». En même temps, les artistes se rendent bien compte que l'invention vient "d'une couche profonde de l'inconscient» et que "l'enfant raconte dans un état presque second ${ }^{6} »$. Mais ils n'accordent pas d'importance exagérée aux significations d'ordre fantasmatique suggérées par les images poétiques; pour eux, il s'agit avant tout d'un matériau à la fraîcheur inimitable et à l'efficacité prodigieuse quand il s'agit d'étonner et d'émouvoir.

Les créations de Catherine Dasté sont présentées à la Biennale de Venise en I968 et en 1969. Elles y suscitent beaucoup d'enthousiasme : certains créateurs italiens adoptent d'ailleurs immédiatement sa méthode; cependant, de façon intéressante, ils ne vont pas poser au même endroit les limites relatives à l'intervention des enfants. Pour Catherine Dasté, la participation enfantine se limitait à l'invention du matériau poétique, en deçà de toute formalisation dramaturgique et a fortiori scénique. Dans la mesure où les enfants ne parvenaient pas à structurer les histoires, la créatrice française s'était résolue à leur donner elle-même une armature logique : le souci de la cohérence narrative

4. La Tribune de Genève, 9 janvier 1970.

5. Notes de C. Dasté, dans les archives du Théâtre de la Pomme Verte (Société d'histoire du théâtre).

6. La Tribune de Genève, art. cité. 
l'emportait ainsi sur ce qui aurait été un respect mimétique de l'imaginaire enfantin. De plus, dans ses spectacles, les artistes adultes se chargeaient du jeu et de la mise en scène; les costumes, réalisés à partir des dessins des enfants, n'en étaient pas moins élaborés par une technicienne experte. Au fond, il était question de réaliser un compromis entre la fidélité à l'imaginaire des enfants et les exigences esthétiques d'un spectacle théâtral.

De façon plus audacieuse, avec le Teatro del Sole (en 1969) puis le Teatro dell'Angolo (en 1973), les enfants se voient confier les clés de la dramaturgie et de la mise en scène, ne laissant théoriquement aux adultes que le champ de l'interprétation. La città degli animali (La Ville des animaux) est le premier avatar italien de la méthode Dasté : Carlo Formigoni, directeur artistique du Teatro del Sole de Turin, adopte le scénario des élèves d'une école élémentaire sans en changer un mot (en dépit de son canevas passablement désarticulé) et soumet ses comédiens à leurs directives scéniques. Le résultat est par conséquent très inhabituel! Dans le même esprit, les premières créations du Teatro dell'Angolo - $L a$ festa dentro la testa I e 2 (La Fête dans la tête I et 2) - assument les incohérences narratives et substituent une composition éclatée, protéiforme, aux règles traditionnelles de la structure linéaire. Aux yeux des créateurs italiens, le succès de ces spectacles définit un nouvel horizon pour le théâtre à venir : plus qu'un "produit fantastique», ce dernier doit constituer une "provocation fantastique ${ }^{7}$ ».

Quand, en 1974, l'écrivain Gianni Rodari collabore avec le Teatro Aperto '74 (une compagnie de La Spezia), il semble redécouvrir pour son compte la méthode Dasté : en effet il recourt à diverses techniques pour faire inventer des histoires aux enfants (proches de celles qu'il expose dans sa Grammaire de l'imagination $^{8}$ ) tout en se réservant le champ de la dramaturgie proprement dite. Loin de mettre en exergue son talent d'auteur, il le met au service d'une communication théâtrale entre enfants : avec La storia di tutte le storie (L'histoire de toutes les histoires), son but est de leur « restituer [...] sous forme de représentation le monde auquel ils [ont] su donner vie, mais auquel ils n'auraient pas su donner forme ${ }^{9}$ ». Toutefois sa fidélité à l'imaginaire des jeunes est moins littérale qu'interprétative : à travers les péripéties merveilleuses du voyage qu'ils racontent, il déchiffre les étapes symboliques du trajet que chacun accomplit pour grandir. Comme Catherine Dasté, il prend soin de sélectionner les éléments clés des récits enfantins, mais c’est pour mieux en révéler les ressorts psychologiques inconscients.

7. Animazione e città, ouvr. cité, p. 21 : "prodotto fantastico", "provocazione fantastica".

8. G. Rodari, Grammaire de l'imagination, Paris, Rue du Monde, 1997.

9. E. Luzzati, G. Rodari, Teatro Aperto '74, Il teatro, i ragazzi, la città, Milan, Emme, 1978. Extrait de "Come nasce un testo teatrale». 
Ainsi, à partir de la méthode Dasté, adoptée et transformée ensuite par les artistes italiens, émerge une nouvelle idée du théâtre pour l'enfance. L'animation a démontré son efficacité en matière de production de spectacles qui, issus de la pensée enfantine, sinon structurés par elle, trouvent naturellement écho auprès du jeune public auquel ils s'adressent. Constitué de récits merveilleux (décousus ou... recousus), ce théâtre récuse toute prétention littéraire mais revendique, à côté de ses enjeux esthétiques, une ambition inédite qu'on pourrait qualifier d'anthropologique. Comédien du Teatro dell'Angolo, Nino d'Introna compare ainsi la démarche artistique de sa compagnie à celle de Peter Brook travaillant avec les tribus africaines : pour les artistes animateurs, l'essentiel n'est pas tant de jouer devant des enfants que de trouver avec eux l'énergie conduisant à la réalisation d'un spectacle ${ }^{\mathrm{ro}}$.

\section{Enjeux éthiques et esthétiques de l'animation}

L'animation théâtrale apparaît comme un ferment de régénération artistique et sociale. Introduite à l'école, elle n'est pas mise au service de la pédagogie institutionnelle mais se veut émancipatrice et subversive. Bien évidemment, le phénomène s'accuse après mai 1968. Chez les artistes engagés, le désir de renouveau théâtral est très vif : leur objectif est de s'adresser prioritairement aux "non-publics», c'est-à-dire à toutes les catégories sociales marginalisées sur le plan culturel; dans ce contexte, créer pour les jeunes semble une voie neuve et pertinente.

Ayant vécu en autarcie durant la période fasciste, l'Italie accuse un retard culturel important et l'institution scolaire, comme le théâtre, y sont en crise. Plus qu'en France, l'animation va susciter l'espoir d'un bouleversement dont la portée pourrait être révolutionnaire. En Italie du Nord, des hommes et des femmes de théâtre - à l'instar de Giuliano Scabia ou de Franco Passatore quittent l'institution théâtrale après 1968 pour trouver des terrains d'exploration davantage en prise avec la société contemporaine. Tournant résolument le dos à la production de spectacles, ils découvrent dans l'école un terrain favorable à l'expérimentation et y rejoignent des enseignants gagnés à la cause de l'animation, tels Remo Rostagno et Loredana Perissinotto. La ville de Turin catalyse le phénomène. Les diverses expériences qui y voient le jour en quelques années ouvrent la voie à un "théâtre des enfants» qui récuse la mystification du

Io. Voir J. Pigeon, Théâtre en mouvement, la création théâtrale pour les jeunes spectateurs dans l'espace européen, Lyon, Les Cahiers du Soleil Debout, I990, p. I7. 
«théâtre pour enfants» et considère que l'acte théâtral véritable s'accomplit non pas dans une réception consommatrice, mais dans un "faire» émancipateur.

À cette époque, un texte de Walter Benjamin, paru en Allemagne en I93I, est traduit et publié en Italie et en France: Pour un théatre d'enfants prolétarien. Il offre une analyse théorique du travail théâtral mené par la comédienne lettone Asja Lacis avec les enfants traumatisés et les orphelins de guerre après la Révolution russe. Dans les deux pays, le texte de Benjamin prend une valeur de manifeste pour un théâtre en train de se construire, en quête de légitimation. Cependant, en France, il paraît alors que les créateurs se sont déjà engagés dans la direction d'un théâtre joué par des adultes à l'intention d'un public d'enfants. Ils ne peuvent prendre au mot le philosophe allemand dans la mesure où celui-ci fait l'apologie d'un théâtre des enfants, «le seul théâtre utilisable pour l'enfant spectateur ${ }^{\mathrm{II}} »$. En revanche, parmi les futurs inventeurs du teatro ragazzi $i^{2}$, nombreux sont ceux qui se reconnaissent littéralement dans le texte de Benjamin. Parmi les expériences marquantes de l'animation italienne, on retiendra particulièrement celles du Teatro Gioco Vita (Théâtre Jeu Vie), désireux d'inventer un théâtre où chacun est " auteur et interprète de lui-même" et où tous jouent "de façon permanente et spontanée leur propre théâtre de vie ${ }^{13} »$. À la place des traditionnelles représentations, la compagnie imagine des fêtes populaires qui rappellent les origines du théâtre lorsque son sens profond était lié aux rituels de la communauté. Franco Passatore invente le concept de "spettacolazione $e^{\mathrm{T}}$ », une action théâtrale improvisée par les enfants qui, s'appuyant sur le travail réalisé en classe avec l'animateur, le redéploient à l'extérieur dans un contexte festif. L'événement peut durer une journée entière et s'effectue à partir d'une structure temporaire que les enfants construisent avec les animateurs. Ayant choisi un thème à mettre en jeu, ils réalisent collectivement la scénographie et les costumes; enfin chacun choisit son rôle et agit librement à l'intérieur de la structure, tandis que les animateurs proposent des accompagnements sonores pour stimuler le jeu. Le soir, s'organise une parade qui permet d'élargir la fête à tout le quartier. Ainsi germe l'utopie d'un théâtre qui ne s'arrêterait jamais, garanti par un espace où

II. W. Benjamin, «Programme pour un théâtre d'enfants prolétarien», trad. P. Ivernel dans A. Lacis, Walter Benjamin et le théâtre pour enfants prolétarien, Strasbourg, Le Portique, 2007. I2. "Théâtre-enfants" : expression qui désigne, depuis ses origines, le théâtre jeune public en Italie.

I3. F. Passatore, S. Destefanis, A. Fontana, F. De Lucis, Io ero l'albero, (tu il cavallo), Florence, Guaraldi Editore, 1972, p. II : "ogni individuo è autore e interprete di sé stesso", "giocare permanentemente e spontaneamente il proprio teatro di vita".

I4. Spettacolazione est un mot-valise étonnant constitué des termes spettacolo («le spectacle»), azione («l'action») et sans doute colazione («le goûter»). 
vie et théâtralité se confondent. Le théâtre n'y est pas représentation, séparant les acteurs des spectateurs, mais acte permanent par lequel la société se donne à elle-même le spectacle de sa propre effervescence créative.

Avec Loredana Perissinotto, l'animation prend une dimension politique forte. Intervenant plusieurs années consécutives dans le quartier Vallette de Turin, elle revendique sans ambiguïté l'instrumentalisation du théâtre à des fins psychologiques et sociales. En 1971, sa première création - La mia, la tua, la sua, la nostra, la vostra, la loro (vita) - est d'inspiration brechtienne : partant des préoccupations des jeunes (la drogue, les conflits familiaux...), l'action présentée débouche sur un débat avec le public. Par la suite, Loredana Perissinotto évolue vers l'agit-prop : en 1972, la dramatisation Homexchelitepilenex est un véritable happening au cours duquel les adolescents, abordant les problèmes de la consommation et de l'écologie, cherchent concrètement à impliquer le public dans leurs paroles et leurs actions de contestation.

Au début des années 1970, l'animation croit avoir devant elle un avenir incommensurable et son idéologie se radicalise : ses plus ardents défenseurs proposent même de se débarrasser du théâtre officiel pour fonder un théâtre communautaire autosuffisant. L'animation déboucherait, en dernière instance, sur une civilisation théâtrale autonome et heureuse où chacun se saisirait de l'art de façon démocratique, sans privilège élitiste, fût-ce au nom du génie personnel. Cependant pour qu'un théâtre jeune public existe, il était nécessaire que l'adulte reprît ses responsabilités de créateur de sorte que les enjeux éthiques émergés durant la période de l'animation pussent trouver leur juste expression sur le terrain esthétique. Pendant une parenthèse utopique de quelques années, les animateurs italiens ont été sourds à cet argument et ont privilégié l'expérience au détriment de l'œuvre.

La Biennale de Venise est alors le lieu d'exhibition des tensions entre les animateurs et les professionnels du théâtre pour enfants. Les spectacles issus de l'animation semblent plus novateurs aux yeux des observateurs de l'époque mais ils constituent des objets théâtraux paradoxaux, inextricablement liés aux lieux et aux circonstances qui leur donnent vie. Face à cet état de crise, le professeur Gian Renzo Morteo va s'imposer comme une figure conciliatrice : il convainc peu à peu les artistes du fait que l'animation doit être considérée comme une propédeutique à la création. "Si on a cultivé un arbre, dit-il, et qu'on l'a cultivé, taillé, fertilisé, il faut savoir reconnaitre que les pommes sont mûres et les cueillir, plutôt que les jeter ${ }^{15}$.» Autrement dit, l'animation constitue

15. Voir M. Bianchi, Atlante del teatro ragazzi in Italia, Corazzano, Titivillus Edizioni, 2009, p. 29 : "Se si pianta un albero, si coltiva, si pota, si concima, quando le mele sono mature si raccolgono, ma non si buttano via." 
un préalable historique essentiel qui rend possible l'élaboration imminente d'un théâtre pour les jeunes.

En France, le débat ne se pose pas dans les mêmes termes. Dès le festival d'Avignon 1969, avec les "Journées du jeune spectateur", émerge avec clarté l'idée d'un théâtre pour la jeunesse joué par des comédiens adultes. Contrairement au cas italien, le festival n'est pas un territoire de conflits pour les protagonistes du domaine; il offre l'occasion de mettre en lumière des formes nouvelles de création et de communication en direction de la jeunesse. Les trois compagnies programmées «tentent d'instituer, en France, un théâtre spécifiquement conçu pour les enfants ${ }^{16} »$ : le Théâtre de la Clairière (de Miguel Demuynck), la Pomme Verte (de Catherine Dasté) et le Théâtre des Jeunes Années (de Maurice Yendt). Avoir choisi le terme de «théâtre pour les jeunes spectateurs" n'est pas anodin : il s'agit de conférer de la dignité au regard posé par l'enfant sur le phénomène théâtral.

Les trois spectacles présentés sont autant de modèles pour ce théâtre à venir. $S$ 'ils semblent de prime abord assez différents, ils ont ceci de commun qu'ils tournent le dos au théâtre de participation, dont l'influence était dominante jusqu'alors lorsqu'on s'adressait à un jeune public : dans un tel théâtre, les enfants étaient amenés à prendre part à l'action dramatique à certains moments clés du spectacle en prenant fait et cause pour le personnage principal. Ce mode d'intervention des enfants spectateurs, très entraînant pour eux, est tenu à distance par les trois compagnies protagonistes du festival. En revanche, chacune intègre à sa manière le concept d'animation.

Deux d'entre elles ne renoncent pas à donner à l'enfant spectateur une marge de manœuvre à l'intérieur du spectacle; toutefois elles ne situent pas l'interaction au même niveau. Pour Catherine Dasté, celle-ci se situe en amont, l'animation constituant la phase fondamentale du processus de création : L'Arbre sorcier, Jérôme et la tortue est un "scénario" cousu à partir d'histoires enfantines, la mise en scène cherchant à donner pleine mesure expressive à l'imaginaire de l'enfant. Miguel Demuynck, quant à lui, développe l'échange avec les enfants durant le temps de la représentation théatrale. Avec Le Pêcheur d'images, le Théâtre de la Clairière privilégie l'expression corporelle et donne vie aux songeries d'un pêcheur; mais bien vite l'image d'un spectacle préalablement construit se défait et les jeunes spectateurs sont invités à émettre des critiques ou à suggérer des thèmes sur lesquels les comédiens se mettent à improviser. Cependant Miguel Demuynck fait en sorte de maintenir les enfants en position de spectateurs critiques, sans jamais leur demander de prendre la place des comédiens (à la différence de ce qui se passe dans le théâtre-forum).

16. Guide d'Avignon, 1969. 
Lors des interruptions internes à la représentation, les acteurs prennent en compte immédiatement les remarques des jeunes en modifiant leur jeu comme s'ils réagissaient aux "retours" d'un metteur en scène. Le but est d'amener le public à prendre du recul face à la fiction. En effet, ainsi que l'exprime Bernard Dort, "la liberté du spectateur [...] ne saurait être d'entrer dans le spectacle»; il faut au contraire «rompre son identification à un personnage», lui faire "reconn[aître] le caractère ludique et fictionnel de la représentation " afin qu'il puisse «devenir le plus spectateur possible ${ }^{17}$ ».

Ainsi comprise, l'animation apparaît comme une alternative salvatrice visà-vis d'un théâtre de participation jugé peu compatible avec l'émancipation de l'enfant spectateur. À première vue, le Théâtre des Jeunes Années semble se situer en décalage avec les deux autres compagnies car il propose un spectacle «adulte» de part en part. Avec Le Pays du soleil debout, un texte dont il est l'auteur, Maurice Yendt dévoile en effet son intention naissante : affirmer une écriture originale en partant du principe que le théâtre pour les jeunes est «un champ totalement vierge de la dramaturgie. " Pourtant, lui aussi se considère comme un «spécialiste» du "dialogue théâtral avec les publics d'enfants ${ }^{18}$ ». Mais il veut repousser ce dialogue hors des cadres de la représentation; menées après le spectacle, les séances d'animation doivent permettre de comprendre après-coup, notamment par le biais du jeu dramatique, ce qui a été vraiment reçu par le jeune public et ainsi de mieux connaître ses besoins en matière de théâtre.

Chacune à sa manière, les trois compagnies d'Avignon tirent donc de l'animation une méthodologie en vue de créer un nouveau théâtre pour les jeunes : quoique instituant une barrière claire entre scène et salle, entre créateurs et destinataires, ce théâtre revendique le besoin de ménager une ouverture, de prévoir un interstice susceptible de créer du jeu et de permettre une remise en cause féconde. Partant de la reconnaissance d'un nouveau public (celui des enfants, vus comme des spectateurs et des individus à part entière), il se conçoit désormais comme un théâtre de recherche au sein duquel l'enfant spectateur doit jouer un rôle actif. Ce rôle ne saurait se réduire à la participation (qui n'est qu'un ersatz d'action) : l'enfant doit être amené à se positionner face à la création, dans une perspective elle-même créative (dans le cas de Catherine Dasté) ou critique (dans les deux autres).

I7. B. Dort, Le spectateur en dialogue, Paris, P.O.L, 1995, p. 96.

I8. M. Yendt et al., Théâtre des Jeunes Années. Une scène de conquête et d'innovation, Carnières, Lansman, 2009, p. 20 et p. 322. 


\section{Vers un théâtre pour les jeunes}

Ce théâtre pour les jeunes issu de l'animation révèle des créateurs qu'on qualifiera de "spécialistes» : ayant pris soin d'établir un rapport avec les enfants en dehors des frontières strictes de la représentation, ils ont pu acquérir, a priori de la mise en œuvre artistique, une conception adéquate de la réalité enfantine (sociale autant que psychologique) susceptible de les aider à inventer des formes ou des contenus dramatiques adaptés. Au cours des décennies 1980-1990, le théâtre jeune public va d'ailleurs se développer en France et en Italie (avec la création, dans les deux pays, de centres dramatiques nationaux pour l'enfance et la jeunesse) sous l'impulsion des compagnies qui s'étaient impliquées dans l'animation théâtrale : la Pomme Verte (initialement conduite par Catherine Dasté) et le Théâtre des Jeunes Années (codirigé durant plusieurs décennies par Maurice Yendt et Michel Dieuaide) comptent parmi les toutes premières structures françaises, de même qu'en Italie le Teatro dell'Angolo ou le Teatro Gioco Vita.

Toutefois, à la suite de ce premier mouvement, émerge en France un contremodèle intéressant. Il s’incarne de façon précoce, en 1973, dans l'éphémère Théâtre National des Enfants : lorsqu'Antoine Vitez, metteur en scène patenté, prétend inventer un théâtre pour les jeunes sans prendre en compte la spécificité du destinataire en se référant à un absolu de l'art, il fait chanceler l'hypothèse fondatrice d'un théâtre spécialisé, garant des intérêts culturels de l'enfant. À propos de Vendredi ou la vie sauvage, spectacle qu'il élabore d'après le roman de Tournier, il déclare : "Au lieu de partir de ce qu'on croit être la vision des enfants, nous créons en toute souveraineté un poème scénique composé d'images et d'événements et nous prenons le pari que les enfants "décolleront" et rêveront à partir de la violence et de la précision des images érotiques ${ }^{19}$."

Quoique sa mise en scène emprunte lisiblement aux mythologies de l'enfance, Vitez se refuse à toute concession sur le terrain du symbolisme : ainsi sur scène il n'y a pas un, mais trois Robinsons, incarnant chacun une étape de la trajectoire existentielle du personnage; les actrices, quant à elles, sont invitées à jouer la part féminine de l'île dans un défoulement de sensualité très suggestif. Enfin le spectacle dure près de deux heures, ce qui est peu adapté au public enfantin. Dans ces conditions, le Vendredi de Vitez ne reçoit qu'un accueil mitigé. Il faudra attendre les créations de Joël Jouanneau (Mamie Ouate en Papoâsie, en 1988) ou d'Olivier Py (La Jeune Fille, le Diable et le Moulin, en 1993) pour que des artistes non spécialistes deviennent véritablement partie

19. Le Monde, avril 1973. 
prenante de l'invention d'un théâtre pour les jeunes. Le Centre dramatique de Sartrouville, Heyoka, œuvre alors consciemment dans cette direction.

Avec le temps, le rôle de l'animation s'est effacé à mesure que celui des metteurs en scène et des auteurs prenait de l'ampleur. La dichotomie entre «spécialistes» et "non spécialistes» s'est assouplie parce que les premiers ont appris à tenir à juste distance leur destinataire d'élection tandis que certains, parmi les seconds, se sont mis à créer régulièrement en direction des jeunes au point de devenir des «habitués» du secteur. Aujourd'hui, l'ambition éthique portée par l'animation s'est-elle étiolée? Elle a pu parfois se diffuser dans les contenus fictionnels du théâtre contemporain pour la jeunesse. En France, où l'édition a joué un rôle majeur, certains textes d'auteurs témoignent d'un véritable engagement social et politique auquel les jeunes peuvent être sensibles : ainsi les pièces de Sylvain Levey (Cent culottes et sans papiers) ou de Catherine Zambon (Mon frère, ma princesse) ont-elles reçu le prix Collidram, prix collégien de littérature dramatique, en 2011 et en 2013.

Si en Italie le répertoire dramatique est loin d'avoir connu le même développement qu'en France, les compagnies les plus renommées ont axé leur recherche sur la spécificité de la réception enfantine. C'est le cas du Teatro delle Briciole (compagnie pionnière du teatro ragazzi) ou encore de la Societas Raffaello Sanzio (compagnie non spécialiste des Castellucci et Guidi). En approfondissant la dimension perceptive de leurs spectacles - dans Buchettino, la Societas Raffaello Sanzio travaille intensément les composantes sonores pour que chaque enfant, couché dans un petit lit, se forge ses propres images à partir d'elles - ou en garantissant l'immersion des jeunes spectateurs dans la fiction théâtrale - Con la bambola in tasca, par les Briciole, fait d'une enfant spectatrice l'héroöne de l'histoire ${ }^{20}$ - elles sont parvenues à une maîtrise éclatante de la relation avec le public qui dépasse, sans les renier, les objectifs que s'était fixés l'animation théâtrale.

20. Buchettino (Le Petit Poucet) et Con la bambola in tasca (La Poupée dans la poche) datent de 1994. 